\title{
Association between trauma and acute hemorrhage of cavernous malformations in children: report of 3 cases
}

\author{
Andrew A. Fanous, MD, 1,2 Patrick K. Jowdy, MD,, ${ }^{1,2}$ Lindsay J. Lipinski, MD,, ${ }^{1,2}$ Lucia L. Balos, MD, ${ }^{3}$ \\ and Veetai $\mathrm{Li}, \mathrm{MD}^{1,2}$ \\ ${ }^{1}$ Department of Neurosurgery, Jacobs School of Medicine and Biomedical Sciences, University at Buffalo, State University of \\ New York; ${ }^{2}$ Department of Neurosurgery, Women \& Children's Hospital of Buffalo-Kaleida Health; and ${ }^{3}$ Department of Pathology \\ and Anatomical Sciences, Kaleida Health System; Buffalo, New York
}

\begin{abstract}
OBJECTIVE Cavernous hemangiomas are benign congenital vascular abnormalities. Intracerebral cavernous hemangiomas have an appreciable risk of spontaneous hemorrhage. Little is known as to whether head trauma increases the risk of bleeding for these lesions. In this study, the authors present a case series of 3 patients with posttraumatic nonspontaneous hemorrhage of intracerebral cavernous malformations (CMs). For the first time, to the authors' knowledge, they propose that trauma might constitute a risk factor for acute hemorrhage in intracerebral cavernomas.

METHODS The authors reviewed the charts of all patients with a new diagnosis of intracerebral cavernoma at their pediatric hospital between 2010 and 2014. Patients with a history of head trauma prior to presentation were subsequently studied to identify features common to these posttraumatic, hemorrhage-prone lesions.
\end{abstract}

RESULTS A history of head trauma was identified in 3 of 19 cases. These 3 patients presented with seizures and/or headaches and were found to have acute hemorrhage within a cavernous hemangioma. None of these patients had any history of abnormal neurological symptoms. All 3 abnormal vascular lesions had associated developmental venous anomalies (DVAs). The 3 patients underwent resection of their respective vascular abnormalities, and the diagnosis of cavernous hemangioma was confirmed with postsurgical tissue pathology. All 3 patients had complete resolution of symptoms following complete excision of their lesions.

CONCLUSIONS Trauma may represent a risk factor for acute hemorrhage in patients with CMs. The presence of associated DVAs may represent a risk factor for posttraumatic hemorrhage of cavernomas. Excision should be considered in such cases, if feasible.

http://thejns.org/doi/abs/10.3171/2016.3.PEDS15517

KEY WORDS acute hemorrhage; cavernous malformation; developmental venous anomaly; traumatic brain injury; trauma; vascular disorders

$\mathrm{C}$ AVERNOUS malformations (CMs), also known as cavernous hemangiomas or cavernomas, are benign vascular lesions that can occur in many different organs, including those of the central nervous system. They consist of abnormally thin-walled and enlarged capillaries that lack elastic tissue. In addition, they are known to be devoid of any intervening parenchyma or any feeding vessels. ${ }^{7}$ On imaging studies, CMs are characterized by the presence of blood products of different ages. In the brain, these abnormal vascular lesions tend to be asymptomatic, and more than $40 \%$ of all CMs are found incidentally on
MRI. ${ }^{4}$ Symptomatic patients with CMs may present with seizures, neurological deficits, or headaches. ${ }^{15,22}$ The diagnosis of a CM is established with the aid of MRI, which is both sensitive and specific. ${ }^{7,22,26}$

The risk of spontaneous hemorrhage from a $\mathrm{CM}$ ranges between $0.4 \%$ and $2.0 \%$ per year for incidentally discovered, previously unruptured cavernomas and between $4.5 \%$ and $22.9 \%$ in CMs with previous hemorrhage. ${ }^{3,14,19,24,25,27}$ Hemorrhage of these lesions is clinically significant in more than $50 \%$ of cases, resulting in headaches, neurological deficits, seizures, and, at times, intractable epilep-

ABBREVIATIONS $\mathrm{CM}=$ cavernous malformation; $\mathrm{CTA}=\mathrm{CT}$ angiography; $\mathrm{DVA}=$ developmental venous anomaly; $\mathrm{WCHOB}=$ Women and Children's Hospital of Buffalo SUBMITTED August 24, 2015. ACCEPTED March 2, 2016.

INCLUDE WHEN CITING Published online May 6, 2016; DOI: 10.3171/2016.3.PEDS15517. 
sy. ${ }^{15,26}$ Risk factors that contribute to an increased threat of spontaneous hemorrhage include size and location of the lesion, previous hemorrhage, and association with a developmental venous anomaly (DVA)..$^{3,4,8,10,14,23}$ On the other hand, reports of nonspontaneous posttraumatic hemorrhage of CM are scarce. ${ }^{5,6}$

We report a series of 3 patients who presented to our institution with posttraumatic nonspontaneous CM hemorrhage. We present details of the clinical course for each patient and describe certain features common to all 3 cases. In addition, we provide a review of the literature for all reports of posttraumatic rupture of intracerebral CMs.

\section{Methods \\ Background}

After receiving institutional review board approval, we reviewed the charts of all patients with a new diagnosis of intracerebral cavernoma at our pediatric hospital (Women and Children's Hospital of Buffalo [WCHOB]) treated between January 1, 2010, and January 1, 2014. Inclusion criteria for our case series was acute hemorrhage of an intracerebral CM following head trauma. Thus, patients with a history of head trauma prior to presentation were studied to identify features common to these posttraumatic, hemorrhage-prone lesions. Patients with a newly diagnosed CM but no inciting traumatic event were excluded from our case series.

\section{Findings}

Nineteen patients (average age 6 years) with newly diagnosed CMs were seen at our institution within the last 4 years. In most of these cases, namely 16 of 19, no history of trauma was reported. In these cases, the vascular abnormality was identified during investigation of nontraumatic symptoms, most commonly seizures and headaches. The diagnosis of CM in these 16 patients was confirmed with MRI. Only 3 patients suffered an acute hemorrhage of intracerebral CM immediately after a traumatic injury to the head. All 3 patients were completely asymptomatic before the injury, suggesting that trauma may have been the trigger for the clinically significant hemorrhage.

\section{Case Series}

Case 1

Presentation and Examination. This 9-year-old boy presented to the emergency department at WCHOB with headaches after a ladder struck him on the right side of his head. He did not lose consciousness. A CT scan demonstrated a right parietal hyperdensity consistent with acute hemorrhage (Fig. 1A). However, the hemorrhage was unusual because it contained small foci of calcification, and its location was atypical for trauma-induced hemorrhage. Brain MRI (Fig. 1B) and MR angiography confirmed the presence of hemorrhage and calcification, suggestive of a CM. These studies also suggested the presence of a dilated venous structure adjacent to the hemorrhage, consistent with a DVA. The presence of the DVA was confirmed with CT angiography (CTA) (Fig. 1C).

Observation and Imaging Findings. The patient re- mained in stable condition and was neurologically intact. Options of surgical intervention versus observation were discussed with the parents, who chose the latter. Subsequent imaging of the brain obtained at various intervals up to 6 months revealed stable appearance of the right parietal abnormality. However, MRI performed 10 months after the patient's initial presentation revealed new and resolving hyperintense signals in the anterior aspect of the lesion on both T1- and T2-weighted sequences, suggestive of subacute hemorrhage (Fig. 1D). Clinically, the patient complained of infrequent low-grade headaches, which also coincided with deterioration of his academic performance.

Operation. Because of the changes observed on the imaging studies and the yearly risk of spontaneous hemorrhage, the family opted for surgical intervention. The patient underwent a right-sided craniotomy for resection of the vascular abnormality. The DVA was left intact during the procedure.

Postoperative Course and Follow-Up. The diagnosis of CM was confirmed after histopathological examination that revealed the presence of thrombosis, calcification, and hemosiderin-laden macrophages (Fig. 1E). The patient remained neurologically intact. Brain MR images obtained immediately postoperatively as well as at 6- and 12-month intervals revealed a largely stable appearance of the resection cavity. However, MRI performed 18 and 24 months after resection revealed progressive blossoming of a focus within the superior aspect of the resection cavity, which appeared hyperintense on both T1- and T2weighted sequences, suggestive of new hemorrhage. Clinically, this corresponded to an increase in the patient's headache intensity and frequency, as well as worsening memory and school performance.

Second Operation and Follow-Up. In view of both the radiological findings and the clinical symptoms, the family again opted for surgical intervention, and the patient underwent a second right-sided craniotomy for resection of the recurrent CM. Postoperative MRI demonstrated complete resection of the CM. The patient has done well for the 18 months since the surgery. The intensity and frequency of his headaches have decreased significantly, and his academic performance has returned to its initial baseline. He remains neurologically intact.

\section{Case 2}

Presentation and Examination. This 3-year-old healthy girl fell backward while attempting to climb a chair, striking the back of her head against a fireplace. There was no loss of consciousness, but she was noted to be unsteady on her feet. She subsequently proceeded to complain of headache and had 3 episodes of emesis, which prompted evaluation at the WCHOB emergency department. Head CT scans revealed a right parietal lesion with a hyperdense rim suggestive of hemorrhage (Fig. 2A). Brain MRI demonstrated a largely hyperintense lesion with a hypointense rim on both T1- and T2weighted sequences, as well as areas of varied intensities indicative of blood products of various ages, suggestive of a CM (Fig. 2B). Contrast enhancement demonstrated the presence of a venous anomaly adjacent to the area of 

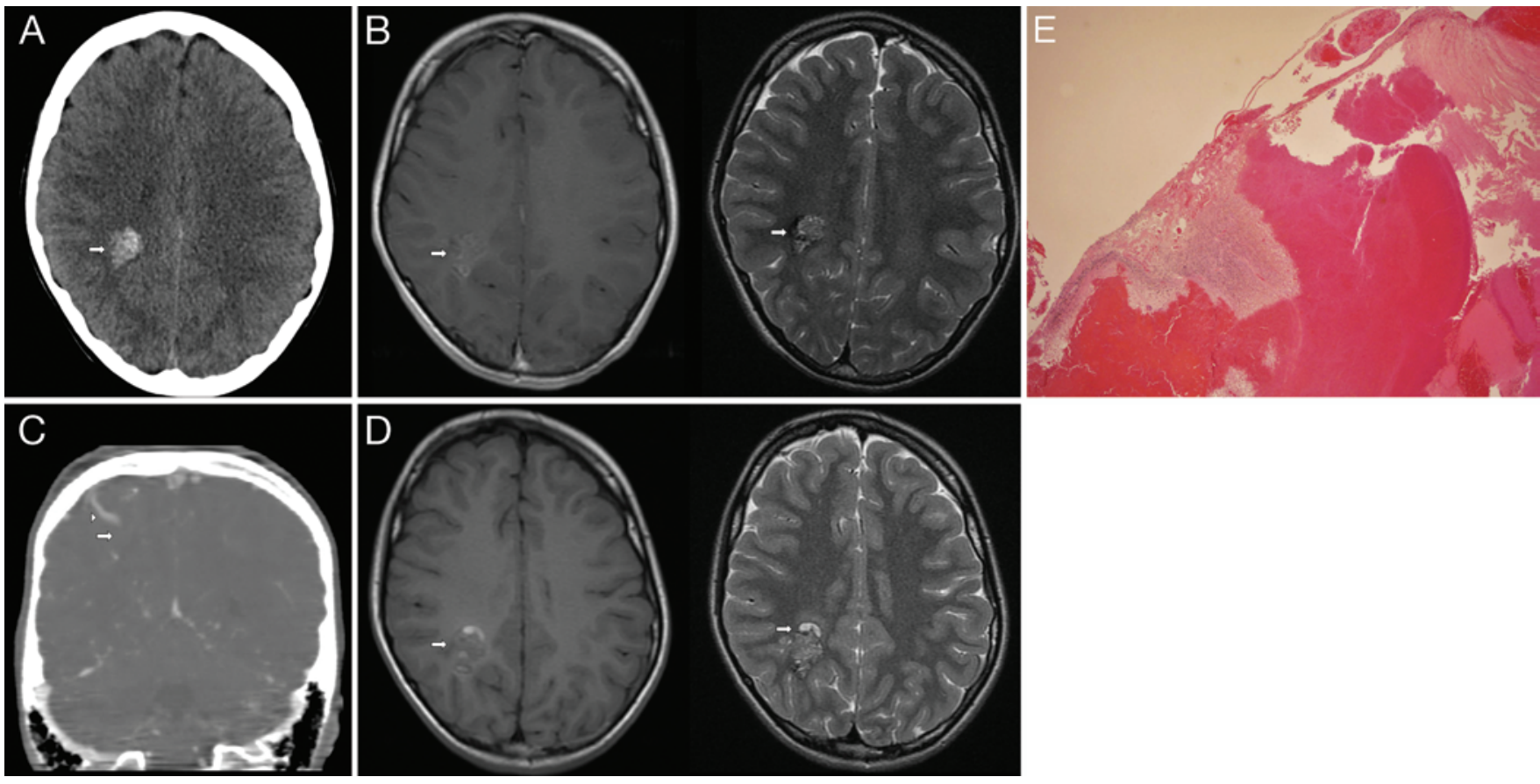

FIG. 1. Case 1. A: Noncontrast axial head CT scan showing a right parietal hyperdensity consistent with acute hemorrhage. B: Axial brain T1-weighted contrast-enhanced (left) and T2-weighted (right) MR images demonstrating blood and calcification at the site of the hemorrhage. C: Head CT angiogram, coronal view, showing the presence of a dilated venous structure adjacent to the hemorrhage. D: Brain MR images obtained at 10 months demonstrating new and resolving hyperintense signals in the anterior aspect of the lesion on both T1-weighted (left) and T2-weighted (right) sequences, suggestive of subacute hemorrhage. Arrows point to the CMs, arrowheads to associated DVAs. E: Photomicrograph of surgically resected lesion confirming the diagnosis of $\mathrm{CM}$. $\mathrm{H} \& \mathrm{E}$, original magnification $\times 20$. Figure is available in color online only.

hemorrhage (Fig. 2C). Serial imaging revealed a stable appearance of the hemorrhage. The patient remained neurologically intact.

Observation and Imaging Findings. Options of surgical intervention versus observation were discussed with the family, who opted for the latter. MRI obtained 2 months after the patient's initial presentation again demonstrated a heterogeneous signal within the lesion, suggestive of hemorrhage of various ages.

Five months after her initial presentation, the patient presented once again to the emergency department at WCHOB after getting struck on the head by a soccer ball. There was no loss of consciousness and no neurological symptoms were present. CT of the head showed a focus of hyperdensity in the area of the CM, suggestive of new hemorrhage (Fig. 2D). The presence of new blood was confirmed by the presence of a hyperintense signal on the T1-weighted sequence and a hypointense signal on the T2-weighted sequence (Fig. 2E). Serial MRI revealed a stable appearance of the hemorrhage, and the patient was discharged home 2 days later. Treatment options were discussed with the family who again opted for expectant observation. However, in the weeks following her discharge, the patient experienced multiple episodes of tonic-clonic seizures that were ultimately controlled by antiepileptic medications. In the subsequent months, she began suffering from frequent headaches and bouts of emesis, as well as irritability. MRI performed 6 months after the patient's second trauma revealed a new area of hyperintensity on the T1-weighted sequence, with corresponding T2 hyper- intensity, which was concerning for new hemorrhage (Fig. 2F).

Operation and Follow-Up. In light of these finding and considering the yearly hemorrhage risk associated with CMs, the family opted for surgical intervention at this time. The patient underwent a right-sided craniotomy for resection of the CM (Fig. 2G). Histopathological examination confirmed the diagnosis of CM. MRI performed 12 months after the surgery revealed no evidence of residual CM. The patient has remained neurologically intact since her surgery 23 months ago, and all her preoperative symptoms have since resolved. She is currently seizure free off antiepileptic medications.

\section{Case 3}

Presentation and Examination. This 17-month-old girl hit the right side of her head against the edge of a table while running. She was initially asymptomatic but developed an acute-onset left-sided gaze deviation and a blank stare 5 hours later. She was nonverbal and unresponsive during this episode. She underwent evaluation in the WCHOB emergency department, where head CT revealed a right occipital focus of acute hemorrhage (Fig. 3A). Brain MRI revealed the presence of a CM underlying the acute blood and comprising both acute and chronic blood products (Fig. 3B). An associated small vessel was identifiable at the superior anterior margin of the lesion (Fig. 3C), likely representing a DVA.

Operation and Follow-Up. In light of the risk of recurrent hemorrhage associated with the $\mathrm{CM}$, the family 

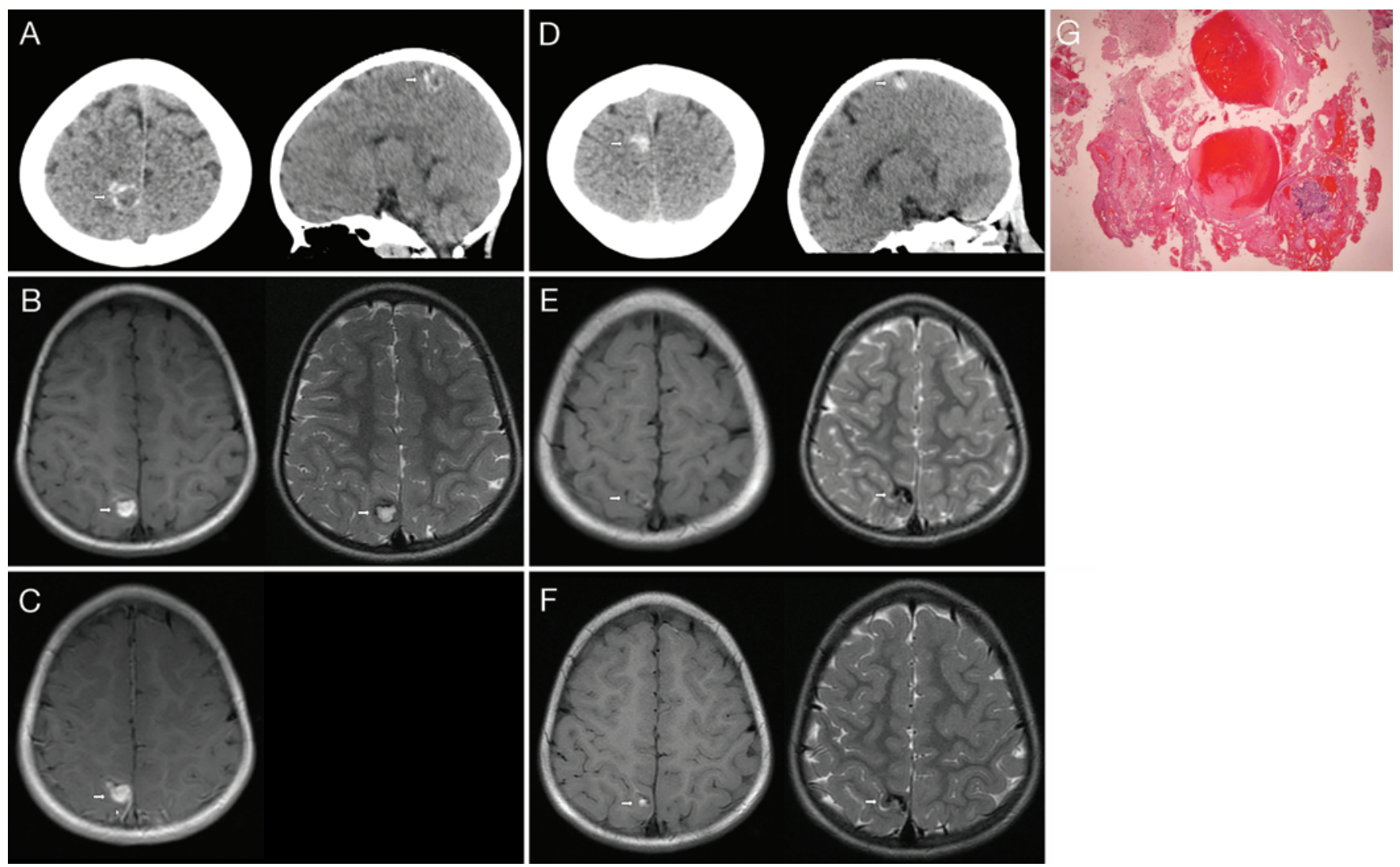

FIG. 2. Case 2. A: Noncontrast head CT scans, axial (left) and sagittal (right) views, demonstrating a right parietal lesion with a hyperdense rim suggestive of hemorrhage. B: Axial T1-weighted (left) and T2-weighted (right) brain MR images demonstrating a hyperintense lesion with a hypointense rim suggestive of a cavernous hemangioma with components of acute hemorrhage. C: Contrast-enhanced T1-weighted MR image demonstrating the presence of a venous anomaly adjacent to the area of the hemorrhagic cavernoma. D: Noncontrast axial (left) and sagittal (right) head CT scans obtained after the second trauma, showing a focus of hyperdensity in the area of the previously identified CM, suggestive of new hemorrhage. E: Axial MR images acquired after the second trauma showing T1 hyperintense signal (left) and T2 hypointense signal (right), also suggestive of new hemorrhage. F: Axial MR images obtained 6 months after the second trauma showing T1 hyperintense signal (left) and T2 hypointense signal (right), again suggestive of new blood. Arrows point to the CMs, arrowheads to associated DVAs. G: Photomicrograph of the resected lesion confirming the diagnosis of $\mathrm{CM}$. $\mathrm{H} \& \mathrm{E}$, original magnification $\times 20$. Figure is available in color online only.

opted for surgical intervention. The patient underwent a right-sided craniotomy and successful resection of the CM (Fig. 3D). Postoperative MRI demonstrated no evidence of residual lesion. The tissue pathology report confirmed the diagnosis of CM. The patient has remained asymptomatic and without focal neurological deficit since her surgery 14 months ago.

\section{Discussion}

Cavernous malformations are a very common intracranial vascular lesion, second only to arteriovenous malformations. ${ }^{10,22}$ Although they are most commonly congenital, they may also develop de novo. ${ }^{21,26}$ The most common presenting feature, seizures, is thought to be caused by the presence of hemosiderin product from remote hemorrhagic events. ${ }^{15,26}$ Spontaneous CM hemorrhage is a wellestablished phenomenon, but reports of trauma-induced hemorrhage are exceedingly rare. The majority of these cases have been described in peritoneal viscera, most notably the liver and the small intestines. ${ }^{1,11-13}$ As noted by Davis et al., ${ }^{9}$ little is currently known about whether head injury increases the risk of bleeding for intracerebral CMs. To our knowledge, there are only 2 reports in the neurosurgical literature that describe posttraumatic hemorrhage of a CM. In one case series comprising 7 patients with posttraumatic intracerebral hemorrhage, fragments of a CM located within the hemorrhage bed were confirmed histologically. ${ }^{5}$ The radiological appearance of these 7 lesions on MRI was never established. The CMs in that case series were most commonly located in the frontal and temporal lobes. There was male sex predominance, and the patients' ages ranged from 33 to 44 years. Cartwright et al. reported the only case of a posttraumatic hemorrhage in an intracerebral CM that was confirmed on MRI. ${ }^{6}$ In that report, a 21-year-old man who was assaulted and suffered facial trauma was later found to have acute hemorrhage within a frontal lobe CM.

In our case series, trauma may have been the inciting event that triggered acute hemorrhage in the CMs in our patients. However, once the initial hemorrhagic event occurred, further bleeding occurred many months later without inciting head trauma. Therefore, there may be an association between trauma and the triggering of the 

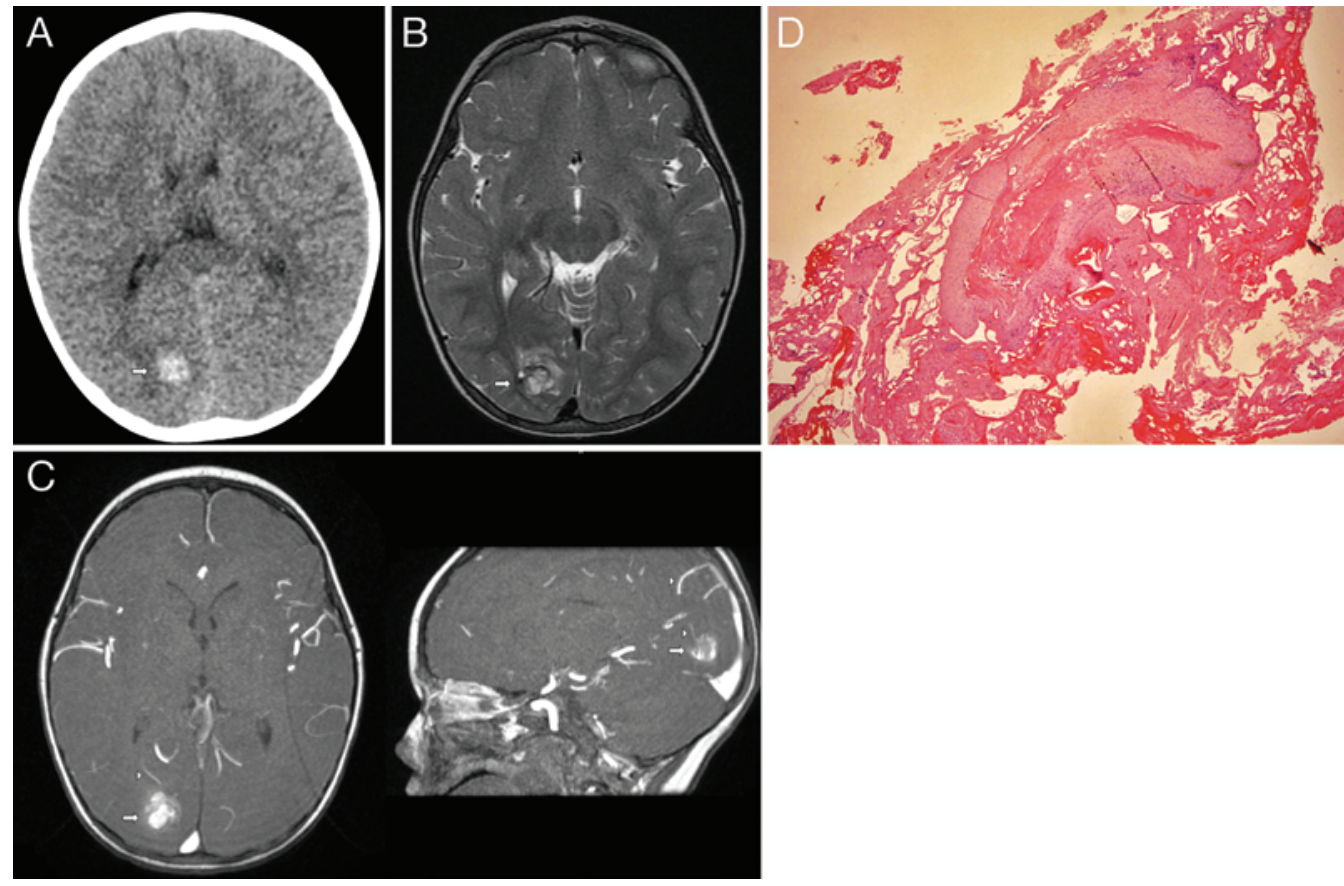

FIG. 3. Case 3. A: Noncontrast axial head CT scan demonstrating a right occipital focus of acute hemorrhage. B: Axial T2weighted MR image demonstrating the presence of a CM comprising acute and chronic blood products. C: Brain MR angiograms, axial (left) and sagittal (right) views, demonstrating the presence of a vascular anomaly at the superior anterior margin of the hemorrhagic cavernoma, possibly representing a deep venous anomaly. Arrows point to the CMs, arrowheads to associated DVAs. D: Photomicrograph of the surgically resected lesion confirming the diagnosis of $\mathrm{CM}$. $\mathrm{H} \& \mathrm{E}$, original magnification $\times 20$. Figure is available in color online only.

bleeding noted immediately after the traumatic episode but not necessarily the bleeding that ensues spontaneously down the road. It is also noteworthy that 2 of our 3 patients had evidence of older blood products on MRI, in addition to the acute blood. This highlights the fact that, although trauma may be one risk factor for acute hemorrhage in $\mathrm{CMs}$, it is by no means the only risk factor, and CMs may still bleed spontaneously without inciting trauma.

The diagnosis of all 3 lesions in this case series was initially established with the help of MRI. The diagnosis was then confirmed postoperatively with histopathological examination. All 3 patients were younger than 10 years of age, reflecting the nature of the patient population at our pediatric trauma center. To our knowledge, this case series is the first to describe an association between trauma and $\mathrm{CM}$ hemorrhage in the pediatric population.

Seizures and headaches were the two most common presenting symptoms in our 3 patients. Symptomatology in all 3 patients correlated with the presence of acute hemorrhage on the imaging studies, and each patient had complete symptom resolution after complete lesion excision. This observation is in agreement with previous observations that have suggested resolution in symptomatology in most patients after excision of CMs ${ }^{15}$ This suggests the importance of surgical intervention in appropriately selected patients with hemorrhagic CMs.

We propose that 2 features of CMs may contribute to the association between acute hemorrhage of these lesions and head injury. First, their characteristically thin walls, which consist of only a single layer of flattened endothelial cells, lack any subendothelial support and contain very few intact tight junctions, may result in the easy rupture of these malformations after trauma. Second and most interestingly, all $3 \mathrm{CMs}$ that hemorrhaged in our case series after trauma had associated DVA. This certainly does not correspond to the rates of CM-associated DVA established in the literature. For instance, in a study of 20 patients with DVAs, Ostertun and Solymosi ${ }^{17}$ reported that only $33 \%$ had an associated CM. On the other hand, in a study by Petersen et al. ${ }^{18}$ of $112 \mathrm{CMs}$, only $8 \%$ had an associated DVA. Abdulrauf et al. ${ }^{2}$ reported the presence of a DVA in only $7.6 \%$ of 55 pediatric patients with CMs. In another study of 37 patients conducted by Revert Ventura et al.,23 only $30 \%$ of the CMs were associated with a DVA. In the same study, the presence of a DVA was associated with a significantly increased risk of spontaneous CM hemorrhage. Other studies have also established the presence of a CM-associated DVA as one of the risk factors for spontaneous nontraumatic hemorrhage of CM..$^{2,3,716,20}$ Thus, a CM associated with a DVA may be more prone to posttraumatic hemorrhage compared with a CM not associated with a DVA. In other words, there may be an association between the presence of a DVA and posttraumatic hemorrhage of a CM. However, larger studies are required to confirm this observation.

As our case series suggests an association between head trauma and hemorrhage of CMs, it may be worthwhile to counsel athletes diagnosed with intracerebral CMs about the potential risks associated with contact sports. Indubitably, a much larger study is required to fully understand the relationship between head injury and acute hemorrhage associated with $\mathrm{CM}$. 


\section{Conclusions}

In our small series, trauma may be a predisposing factor for acute hemorrhage in patients with CM. The presence of an associated DVA may represent a risk factor for posttraumatic hemorrhage of these lesions. Patients with small parenchymal hemorrhagic lesions following head injury should undergo MRI to verify the presence of a CM, particularly if the hemorrhage is atypical for posttraumatic bleeding. Excision may be considered in such cases, provided there is a favorable risk-benefit profile.

\section{Acknowledgments}

We thank Paul H. Dressel, BFA, for preparation of the illustrations and Debra J Zimmer for editorial assistance.

\section{References}

1. Abdul Aziz DA, Khandasamy Y, Tamba RP, Zaki FM: Bleeding small bowel cavernous haemangioma following blunt trauma to the abdomen presenting as subacute intestinal obstruction in a child. BMJ Case Rep 2011:bcr0820114672, 2011

2. Abdulrauf SI, Kaynar MY, Awad IA: A comparison of the clinical profile of cavernous malformations with and without associated venous malformations. Neurosurgery 44:41-47, 1999

3. Aiba T, Tanaka R, Koike T, Kameyama S, Takeda N, Komata T: Natural history of intracranial cavernous malformations. J Neurosurg 83:56-59, 1995

4. Batra S, Lin D, Recinos PF, Zhang J, Rigamonti D: Cavernous malformations: natural history, diagnosis and treatment. Nat Rev Neurol 5:659-670, 2009

5. Beuth W, Woźniak B, Kasprzak H: [Cases of cavernous hemangioma discovered in patients with post traumatic intracerebral hematoma.] Neurol Neurochir Pol 33:603-612, 1999 (Polish)

6. Cartwright G, Bailey BM, Lane RJ, Messiha A: A diagnostic dilemma of intracranial pathology: coincidence or the result of cranial trauma? BMJ Case Rep 2010:bcr0720103167, 2010

7. Coban A, Gurses C, Bilgic B, Sencer S, Karasu A, Bebek N, et al: Sporadic multiple cerebral cavernomatosis: report of a case and review of literature. Neurologist 14:46-49, 2008

8. Cortés Vela JJ, Concepción Aramendía L, Ballenilla Marco F, Gallego León JI, González-Spínola San Gil J: Cerebral cavernous malformations: spectrum of neuroradiological findings. Radiologia 54:401-409, 2012

9. Davis G, Fabinyi G, Le Roux P, McCrory P: Clinics in neurology and neurosurgery of sport. Mass lesions: cavernoma. Br J Sports Med 43:866-868, 2009

10. Gallati CP, Silberstein HJ, Meyers SP: Hemorrhage of a cavernous malformation associated with accidental electrocution: case report and review of the literature. Surg Neurol Int 3:166, 2012

11. Hotokezaka M, Kojima M, Nakamura K, Hidaka H, Nakano Y, Tsuneyoshi M, et al: Traumatic rupture of hepatic hemangioma. J Clin Gastroenterol 23:69-71, 1996

12. Kanazawa S, Douke T, Gotoh A, Nishi A, Yasui K, Kajiwara Y: [A case of giant cavernous hemangioma with hemoperitoneum due to blunt abdominal trauma; CT findings.] Rinsho Hoshasen 35:979-982, 1990 (Jpn)

13. Kocakusak A, Sunar H, Akinci M, Gulen M, Arikan S: [Rupture of an incidental giant liver hemangioma caused by blunt abdominal trauma.] Ulus Travma Derg 8:176-178, 2002 (Turkish)
14. Kondziolka D, Lunsford LD, Kestle JR: The natural history of cerebral cavernous malformations. J Neurosurg 83:820824,1995

15. Moran NF, Fish DR, Kitchen N, Shorvon S, Kendall BE, Stevens JM: Supratentorial cavernous haemangiomas and epilepsy: a review of the literature and case series. J Neurol Neurosurg Psychiatry 66:561-568, 1999

16. Mottolese C, Hermier M, Stan H, Jouvet A, Saint-Pierre G, Froment JC, et al: Central nervous system cavernomas in the pediatric age group. Neurosurg Rev 24:55-73, 2001

17. Ostertun B, Solymosi L: Magnetic resonance angiography of cerebral developmental venous anomalies: its role in differential diagnosis. Neuroradiology 35:97-104, 1993

18. Petersen TA, Morrison LA, Schrader RM, Hart BL: Familial versus sporadic cavernous malformations: differences in developmental venous anomaly association and lesion phenotype. AJNR Am J Neuroradiol 31:377-382, 2010

19. Porter PJ, Willinsky RA, Harper W, Wallace MC: Cerebral cavernous malformations: natural history and prognosis after clinical deterioration with or without hemorrhage. J Neurosurg 87:190-197, 1997

20. Pozzati E, Acciarri N, Tognetti F, Marliani F, Giangaspero F: Growth, subsequent bleeding, and de novo appearance of cerebral cavernous angiomas. Neurosurgery 38:662-670, 1996

21. Pozzati E, Giuliani G, Nuzzo G, Poppi M: The growth of cerebral cavernous angiomas. Neurosurgery 25:92-97, 1989

22. Requena I, Arias M, López-Ibor L, Pereiro I, Barba A, Alonso A, et al: Cavernomas of the central nervous system: clinical and neuroimaging manifestations in 47 patients. J Neurol Neurosurg Psychiatry 54:590-594, 1991

23. Revert Ventura AJ, Martí-Bonmatí L, Poyatos Ruipérez C, Pallardó Calatayud Y, Arana E, Mollá Olmos E: [Association of cavernous and venous angiomas.] Neurologia 22:839-845, 2007 (Span)

24. Robinson JR, Awad IA, Little JR: Natural history of the cavernous angioma. J Neurosurg 75:709-714, 1991

25. Smith ER, Scott RM: Cavernous malformations. Neurosurg Clin N Am 21:483-490, 2010

26. Stefan H, Hammen T: Cavernous haemangiomas, epilepsy and treatment strategies. Acta Neurol Scand 110:393-397, 2004

27. Zabramski JM, Wascher TM, Spetzler RF, Johnson B, Golfinos J, Drayer BP, et al: The natural history of familial cavernous malformations: results of an ongoing study. J Neurosurg 80:422-432, 1994

\section{Disclosures}

The authors report no conflict of interest concerning the materials or methods used in this study or the findings specified in this paper.

\section{Author Contributions}

Conception and design: Fanous. Acquisition of data: all authors. Analysis and interpretation of data: all authors. Drafting the article: Fanous. Critically revising the article: all authors. Reviewed submitted version of manuscript: all authors. Study supervision: Li.

\section{Correspondence}

Veetai Li, Department of Neurosurgery, Women \& Children's Hospital of Buffalo, 219 Bryant St., Buffalo, NY 14222. email: vli@ubns.com. 